\title{
ERRATA
}

\section{GEOMETRIC GALOIS THEORY, NONLINEAR NUMBER FIELDS AND A GALOIS GROUP INTERPRETATION OF THE IDELE CLASS GROUP}

\author{
[Internat. J. Math. 16(6) (2005) 567-593] \\ T. M. GENDRON and A. VERJOVSKY \\ Instituto de Matemáticas \\ Universidad Nacional Autónoma de México \\ Av. Universidad $s / n$, Lomas de Chamilpa \\ Cuernavaca CP 62210, Morelos \\ México
}

Received 22 April 2010

\begin{abstract}
In this errata we correct several mistakes Secs. 1 and 3-6 that appear in the published version of our paper. The corrections have been implemented in the revised version [1]. In addition, in Sec. 2 we clarify an important point which was not adequately addressed in the published version; in Sec. 7 we point out an enhancement of the hyperbolization scheme included in [1]. The reader may also wish to consult [2].
\end{abstract}

Keywords: Nonlinear number field; character field; Minkowski torus; idele class group.

Mathematics Subject Classification 2000: 11R56, 11R37, 11R32

\section{Definition of Nonlinear Number Field}

In the original publication, on page 582 , line 15 , the definition of $\mathbb{C}^{*}[K]$ should read:

$$
\mathbb{C}^{*}[K]=\mathbb{C}[K]-I_{K}
$$

and not " $\mathbb{C}[K] / I_{K}$." The set $(1.1)$ is invariant with respect to both Cauchy and Dirichlet multiplication and the space $\mathbb{C P}[K]$ is then defined to be $\mathbb{C}^{*}[K] / \mathbb{C}^{*}$. This correction must be carried out in the more general definition, e.g. on page 586 , line 30 should read $\mathrm{H}_{\bullet}^{*}[K]=\mathrm{H}_{\bullet}[K]-\mathrm{I}_{K}$, where $\mathrm{I}_{K}$ is the kernel of the trace map on its domain of definition (see $[1$, Sec. 9]).

\section{Dirichlet Product Structure}

Our treatment of the Dirichlet product structure of $\operatorname{Char}\left(\hat{\mathbb{S}}_{K}\right)$ (part 1 of Theorem 5 , page 581) was incomplete. Let $\psi_{\infty}(\mathbf{z})=\exp (2 \pi i \operatorname{Tr}(\mathbf{z}))$ be the standard character on 
$K_{\infty} \subset \hat{\mathbb{S}}_{K}$, which extends continuously to a character $\psi$ on $\hat{\mathbb{S}}_{K}$ that the monomorphism

$$
\alpha \mapsto \psi_{\alpha}, \quad \psi_{\alpha}(\hat{\mathbf{z}})=\psi(\alpha \hat{\mathbf{z}})
$$

defines an isomorphism of additive groups $(K,+) \cong \operatorname{Char}\left(\hat{\mathbb{S}}_{K}\right)$ that is classical [3]. The Dirichlet product $\otimes$ on $\operatorname{Char}\left(\hat{\mathbb{S}}_{K}\right)$ is then defined by pushing forward $\times_{K}$. The crucial observation is that the definition of $\otimes$ is natural with respect to the inclusions $\operatorname{Char}\left(\hat{\mathbb{S}}_{K}\right) \hookrightarrow \operatorname{Char}\left(\hat{\mathbb{S}}_{L}\right)$ induced by the trace maps $\operatorname{Tr}_{L / K}: \hat{\mathbb{S}}_{L} \rightarrow \hat{\mathbb{S}}_{K}$. See [1, Sec. 7] for more details.

\section{Character Group of the Minkowski Torus}

In part 2 of Theorem 5 , the additive group $\left(O_{K},+\right)$ was mistakenly identified with the character group $\operatorname{Char}\left(\mathbb{T}_{K}\right)$ of the Minkowski torus $\mathbb{T}_{K}$ via the map (2.1). Instead, (2.1) identifies the inverse different $\mathfrak{d}_{K}^{-1} \supset O_{K}$ with $\operatorname{Char}\left(\mathbb{T}_{K}\right)$. The character group Char $\left(\mathbb{T}_{K}\right)$ is thus an $O_{K}$-module extension of $O_{K}$ (see [1, Sec. 7$]$ ).

\section{Domain of Definition of Cauchy; Dirichlet Products}

On line 15 , page 583, it was incorrectly asserted that the Cauchy and Dirichlet products are fully defined on the Hilbert space $L^{2}\left(\hat{\mathbb{S}}_{K}, \mathbb{C}\right)$. These operations only extend partially so that for each element $f \in L^{2}\left(\hat{\mathbb{S}}_{K}, \mathbb{C}\right)$ one must specify the Cauchy and Dirichlet domains $\operatorname{Dom}_{\oplus}(f), \operatorname{Dom}_{\otimes}(f)$ of elements $g$ for which $f \oplus g$, respectively $f \otimes g$, makes sense. Note that since the double group algebra $\mathbb{C}[K]$ is contained in $\operatorname{Dom}_{\oplus}(f), \operatorname{Dom}_{\otimes}(f)$, we have that the latter spaces are dense in $L^{2}\left(\hat{\mathbb{S}}_{K}, \mathbb{C}\right)$ (see $[1$, Sec. 8$\left.]\right)$. The definition of nonlinear number field, which appears in Definition 2 on page 587, must be adjusted accordingly by replacing everywhere the phrase "double semigroup" by "partial double semigroup" to take into account this correction (see [1, Sec. 9]).

\section{Proof of Lemma 1}

The proof of Lemma 1 on page 589 was incorrect. Here is a correct proof (which appears as $[1$, Sec. 10, proof of Lemma 2]). Suppose first that $K=\mathbb{Q}$ and without loss of generality $F \in \mathbf{H}[\mathbb{Q}]$. Let $\tau_{0} \in \mathbb{H}_{\mathbb{Q}} \subset \hat{\mathfrak{S}}_{\mathbb{Q}}$ be such that $F\left(\tau_{0}\right) \neq 0$. Then there exists a complex number $\alpha$ with $\exp \left(2 \pi i \alpha \tau_{0}\right)=F\left(\tau_{0}\right)$. By hypothesis, $F(r \tau)=$ $F(\tau)^{r}$, so $F(\tau)$ and $\exp (2 \pi i \alpha \tau)$ agree on the ray $\mathbb{R}_{+} \cdot \tau_{0}$. Then by holomorphicity, they coincide. Since $F$ is a function of $\hat{\mathfrak{S}}_{\mathbb{Q}}$, it follows that $\alpha \in \mathbb{Q}$.

Now let the extension $K / \mathbb{Q}$ be totally real of finite degree. Let $\Delta \subset \mathbb{H}_{K}$ be the diagonal hyperbolic sub-plane (the dense leaf of the image of $\hat{\mathfrak{S}}_{\mathbb{Q}}$ under the diagonal embedding $\hat{\mathfrak{S}}_{\mathbb{Q}} \hookrightarrow \hat{\mathfrak{S}}_{K}$ ). Then by the previous paragraph, the restriction of $F$ to $\Delta$ is an exponential $\exp (2 \pi i q \tau)$ for $q \in \mathbb{Q}$. Let $\Delta_{1} \subset \mathbb{H}_{K}$ be the diagonal $\Delta_{1}=$ $\{(\tau, \ldots, \tau, \tilde{\tau}) \mid \tau, \tilde{\tau} \in \mathbb{H}\} \supset \Delta$. For each $\tau$ fixed, we can (using the same argument 
employed in the previous paragraph) write the function $\tilde{\tau} \mapsto F(\tau, \ldots, \tau, \tilde{\tau})$ as

$$
F(\tau, \ldots, \tau, \tilde{\tau})=\exp (2 \pi i q \tau) \cdot \exp (2 \pi i \beta(\tau) \tilde{\tau})
$$

where $\beta(\tau) \in \mathbb{C}$. Moreover we have

$$
F(r \cdot(\tau, \ldots, \tau, \tilde{\tau}))=\exp (2 \pi i q r \tau) \cdot \exp (2 \pi i \beta(\tau) r \tilde{\tau})
$$

by hypothesis. As we vary $\tau, \beta(\tau)$ varies holomorphically and we obtain that on a real codimension one subspace of $\Delta_{1}$,

$$
F(\tau, \ldots, \tau, \tilde{\tau})=F_{1}(\tau, \ldots, \tau, \tilde{\tau}):=\exp (2 \pi i q \tau) \cdot \exp (2 \pi i \beta(\tau) \tilde{\tau}),
$$

hence they are equal on $\Delta_{1}$. But this means that $F_{1}$ must also satisfy the functional equation $F_{1}(r \cdot(\tau, \ldots, \tau, \tilde{\tau}))=F_{1}(\tau, \ldots, \tau, \tilde{\tau})^{r}$, which implies that $\beta(\tau)=\beta$ is a constant. Inductively, we obtain that $F$ restricted to $\mathbb{H}_{K}$ is an exponential function, and since $F$ is a function of $\hat{\mathfrak{S}}_{K}$, this restriction must be of the form $\boldsymbol{\xi}^{\alpha}=\exp (2 \pi i \operatorname{Tr}(\alpha \cdot \boldsymbol{\tau}))$ for $\alpha \in K_{+}$. The case of a totally complex field extension, that of mixed type, or that of infinite degree, is handled in exactly the same manner.

\section{Idele Class Group Action}

The discussion of the idele class group found on pages 591 and 592 is only valid for $K=\mathbb{Q}$, so that the idele class group $C_{K}$ should be replaced by $C_{\mathbb{Q}}($ see $[1$, Sec. 10$])$.

\section{Hyperbolization}

Apart from implementing the above corrections, the revised version [1] contains an enhancement of the hyperbolization $\mathbb{H}_{K}$ (page 579 and 580 of the original article), in which for each complex place $\mu$, one takes the product of $K_{\mu} \cong \mathbb{C}$ with the complex quarter plane $\{z=x+i y \mid x, y>0\}$, rather than the factor $(0, \infty) \times(0, \infty)$ (see $[1$, Sec. 6]). Furthermore, the $\theta$-holomorphic grading of functions on $\mathbb{H}_{K}$ (described in page 585) has been expanded by a complex sign set along the complex places (this is described in [1, Sec. 8.2]).

\section{Acknowledgments}

We thank McQuillan for having brought to our attention Secs. 1, 3 and 4 above.

\section{References}

[1] T. M. Gendron and A. Verjovsky, Geometric Galois theory, nonlinear number fields and a Galois group interpretation of the idele class group, revised version [arXiv:math/0506519].

[2] T. M. Gendron and A. Verjovsky, Notes on nonlinear number fields [arXiv: 1007.3070v1] [math.NT].

[3] J. T. Tate, Fourier analysis in number fields, and Hecke's zeta-functions, in 1967 Algebraic Number Theory (Proc. Instructional Conf., Brighton, 1965) (Thompson, Washington D.C.), pp. 305-347. 